\title{
Características da gravidez e lactação de mulheres atendidas em um banco de leite humano
}

\author{
Characteristics of pregnancy and lactation of women \\ served in a human milk bank
}

Helena Siqueira Vassimon', Camila Manfredi dos Santos², Janaína Mendes de Oliveira Cossi ${ }^{3}$, Fabíola Pansani Maniglia ${ }^{4}$

${ }^{7}$ Nutricionista na Prefeitura Municipal de Ribeirão Preto

${ }^{2}$ Docente do Curso de Nutrição da Universidade de Franca

${ }^{3}$ Nutricionista

${ }^{4}$ Docente dos Cursos de Nutrição e Enfermagem da Universidade de Franca

Contato: Fabíola Pansani Maniglia - fa_nutricao@hotmail.com

\section{Resumo}

Objetivo: identificar as características da gravidez e da lactação de mulheres que procuraram um banco de leite humano e as razões que motivaram essas mães a recorrerem ao serviço. Métodos: Trata-se de um estudo realizado no Banco de Leite Humano (BLH) da Fundação Santa Casa da Misericórdia de Franca. Foram recrutadas 60 mulheres que responderam a um questionário para a obtenção de informações a respeito da gestação, da amamentação e dos motivos que as levaram ao banco de leite. Resultados: As participantes realizaram o pré-natal em número adequado de consultas e tinham em média $25(6,1)$ anos. Os sintomas gestacionais mais relatados foram náuseas/vômitos (71,7\%) e picamalácia (60\%). A maior aversão alimentar referida foi à ingestão de carnes (42,3\%). Dentre os fatores que levaram as mulheres a buscarem o BLH, destacaram-se o auxílio para estimular a produção do leite ou para doação do mesmo. 58\% das mulheres estavam em aleitamento materno exclusivo e as demais referiram estar aguardando a descida do leite ou estarem distantes dos bebês, que se encontravam no centro de terapia intensiva. Alguns bebês menores de 2 meses receberam água, chás e papas precocemente. Conclusão: As nutrizes recorreram ao BLH por problemas na amamentação, apesar das orientações no pré-natal. Algumas necessitaram de apoio para o estímulo da produção de leite. A importância do BLH vai além de possibilitar o leite às crianças que não puderam ser amamentadas por suas mães, mas também por proporcionar instrução e apoio às lactantes, além do incentivo ao aleitamento materno.

Palavras-chave: Aleitamento materno. Gestação. Bancos de leite.

\begin{abstract}
Objective: to identify the characteristics of pregnancy and lactation of women who sought a human milk bank and the reasons that motivated these mothers to use the service. Methods: This is a study carried out at the Human Milk Bank (BLH) of the Santa Casa da Misericórdia de Franca Foundation. 60 women were recruited who answered a questionnaire to obtain information about pregnancy, breastfeeding and the reasons that led them to the milk bank. Results: The
\end{abstract}


participants underwent prenatal care in an adequate number of consultations and were on average 25 (6.1) years old. The most reported gestational symptoms were nausea /vomiting (71.7\%) and pica (60\%). The greatest food aversion reported was meat intake (42.3\%). Among the factors that led women to seek HMB, the help to stimulate milk production or to donate it stood out. $58 \%$ of the women were on exclusive breastfeeding and the remainder said they were waiting for the milk to drop or were distant from the babies, who were in the intensive care center. Some babies younger than 2 months received water, teas and porridge early. Conclusion: mothers resorted to HMB due to breastfeeding problems, despite prenatal guidance. Some needed support to stimulate milk production. The importance of BLH goes beyond making milk available to children who could not be breastfed by their mothers, but also for providing instruction and support to breastfeeding mothers, in addition to encouraging breastfeeding.

Keywords: Breastfeeding. Pregnancy. Milk banks.

\section{INTRODUÇÃO}

A gravidez é um evento biologicamente natural e especial na vida da mulher, que muitas vezes pode ter sua ocorrência e evolução influenciadas pelo contexto social e cultural em que a mãe está inserida ${ }^{1}$. Além disso, é neste período que muitas dúvidas afligem a mulher, tornando primordial o acompanhamento pré-natal ${ }^{2}$. É durante o acompanhamento profissional especializado que antecede o parto que os problemas futuros podem ser evitados ou minimizados. O uso de suplementos nutricionais, as instruções sobre o parto e sobre a amamentação, devem ser feitas neste momento ${ }^{2}$.

A amamentação é um processo que exige cuidados específicos e, por isso, deve ser estimulada e orientada durante o pré-natal. De acordo com o Ministério da Saúde os bebês devem receber o leite materno de forma exclusiva até o sexto mês de vida e a partir deste momento, devem ser introduzidos os alimentos que complementarão a nutrição3.

A importância do leite materno vai além de suas propriedades nutricionais que são absolutamente equilibradas para a criança. Este leite é também rico em anticorpos que garantem proteção imunológica ao lactente 3 .

Quando a mãe não recebe suporte profissional adequado durante sua gestação, podem acontecer problemas na amamentação que muitas vezes comprometem a sua manutenção ${ }^{4}$. Ingurgitamento, mastite e bico do peito com fissuras são algumas das principais razões para a descontinuidade do aleitamento materno e introdução das fórmulas infantis. Além destes problemas, outras situações podem inviabilizar a 
amamentação, como a prematuridade, na qual a descida do leite é demorada e os bebês prematuros não têm força para a sucção3,5. No entanto, a proteção imunológica para o lactente pode ser garantida por meio de leites doados por outras mães, que são disponibilizados pelos Bancos de Leite Humano (BLH).

O primeiro BLH do Brasil foi implantado em 1943 e se destinava apenas à coleta do leite das mães doadoras, visando atender os casos considerados especiais. Mas com o desenvolvimento do Programa Nacional de Incentivo ao Aleitamento Materno, a implantação da Rede Nacional de Bancos de Leite Humano (REDEBLH) e o aprimoramento das políticas públicas relacionadas, os BLH passaram a exercer um importante papel de orientação e incentivo à amamentação ${ }^{3,6}$. Atualmente a Rede Brasileira de Banco de Leite Humano é considerada a maior e mais complexa do mundo pela Organização Mundial de Saúde (OMS) ${ }^{7}$.

No ano de 2017 foram coletados em todo país 212 mil litros de leite de 183 mil mães doadoras, beneficiando 198 mil bebês prematuros ${ }^{8}$.

A doação de leite materno é estimulada pelo Ministério da Saúde e acontece em serviços de saúde que possuem o Banco de Leite Humano, local onde se realiza a ordenha, pasteurização e armazenamento dos leites, além de orientação especializada sobre amamentação para as mães que enfrentam dificuldades neste processo $^{9}$.

Com base nas informações supracitadas, o presente estudo teve como objetivo identificar as características da gravidez e da lactação de mulheres que procuraram o BLH da Fundação Santa Casa da Misericórdia de Franca, bem como os motivos que as levaram até lá.

\section{MÉTODO}

Trata-se de uma pesquisa realizada no ano de 2011 com lactantes atendidas no Banco de Leite Humano (BLH) da Fundação Santa Casa da Misericórdia de Franca/SP. A pesquisa foi aprovada em seus aspectos éticos e metodológicos pelo comitê de ética em pesquisa da instituição sob o protocolo de número 096/2010.

Foram adotados como critérios de inclusão as lactantes alfabetizadas que passaram pelo BLH durante o período de coleta de dados de 2 meses e que manifestaram seu interesse, assinando o Termo de Consentimento Livre e Esclarecido (TCLE), totalizando 60 mulheres. Foram excluídas da pesquisa lactantes analfabetas ou que recusam a participar da pesquisa. 
Os dados sociodemográficos foram coletados em entrevista e para a obtenção de informações a respeito da gestação, da amamentação e dos motivos que as levaram ao $\mathrm{BLH}$, foi aplicado um questionário não validado, elaborado pelas próprias pesquisadoras, cujas abordagens estão apresentadas a seguir:

Gestação: foram abordados com as mães assuntos relacionados à suplementação de ferro e folato, exposição solar, sintomas associados à gestação (náuseas, vômitos, picamalácia) e doenças desenvolvidas durante a gestação. Investigou-se também sobre os desejos e aversões alimentares durante o período gestacional.

Amamentação: verificou-se quais nutrizes estavam em amamentação, em caso negativo, quais os motivos dos desmame precoce. Investigou-se a inclusão ou não de fórmulas infantis, água, chá e alimentos nos primeiros 6 meses de vida do bebê. Também foi objeto da pesquisa conhecer a visão da lactante quanto à importância da amamentação.

Atendimento no banco de leite: foram levantados os motivos que levaram as mães a procurarem o atendimento do BLH. Foi realizado um acompanhamento do binômio mãe e filho desde o momento da busca pelo BLH até a solução dos problemas que motivaram a procura pelo serviço. Em todos os casos de assistência, registrou-se o manejo realizado e o tempo gasto para a solução do problema.

Após a coleta dos dados os mesmos foram tabulados para que se realizasse a análise quantitativa descritiva por meio da obtenção dos valores absolutos de média e desvio padrão, bem como das porcentagens.

\section{RESULTADOS}

As participantes do estudo eram majoritariamente naturais da cidade de Franca, situada no interior do estado de São Paulo $(n=55 ; 91,7 \%)$ e tinham em média $25(6,1)$ anos. Em relação à escolaridade, nenhuma participante era analfabeta, e a média de tempo de estudo correspondeu a $11(1,7)$ anos.

Na Tabela 1 constam as demais características sociodemográficas das participantes, bem como as informações relacionadas à gestação e ao parto. 
Helena Siqueira Vassimon, Camila Manfredi dos Santos, Janaína Mendes de Oliveira Cossi, Fabíola Pansani Maniglia | Características da gravidez e lactação de mulheres atendidas em um banco de leite humanol

Tabela 1 - Características sociodemográficas e relacionadas à gestação e ao parto ( $n=60)$ - Franca, 2011.

\begin{tabular}{|c|c|c|}
\hline Características & $\mathbf{N}$ & $\%$ \\
\hline \multicolumn{3}{|l|}{ Situação Conjugal } \\
\hline Casada ou em união consensual & 39 & 65,0 \\
\hline Solteira, separada ou divorciada & 21 & 35,0 \\
\hline \multicolumn{3}{|l|}{ Reside com quem } \\
\hline Marido & 39 & 65,0 \\
\hline Pais & 11 & 18,3 \\
\hline Outras pessoas além do esposo e/ou filhos & 10 & 16,7 \\
\hline \multicolumn{3}{|l|}{ Número de filhos } \\
\hline Um & 40 & 66,7 \\
\hline Dois & 13 & 21,7 \\
\hline Três & 3 & 5,0 \\
\hline Mais que 3 & 4 & 6,6 \\
\hline \multicolumn{3}{|l|}{ Gravidez planejada } \\
\hline Sim & 25 & 41,7 \\
\hline Não & 35 & 58,3 \\
\hline \multicolumn{3}{|l|}{ Realização de pré-natal } \\
\hline Sim & 60 & 100,0 \\
\hline Não & 0 & 0 \\
\hline \multicolumn{3}{|l|}{ Tipo de parto } \\
\hline Cesárea & 32 & 53,3 \\
\hline Normal & 28 & 46,7 \\
\hline \multicolumn{3}{|l|}{ Renda familiar suficiente para compras } \\
\hline Sim & 59 & 98,3 \\
\hline Não & 1 & 1,7 \\
\hline \multicolumn{3}{|l|}{ Situação ocupacional } \\
\hline Do lar & 25 & 41,7 \\
\hline Sapateira & 17 & 28,3 \\
\hline Estudante & 5 & 8,3 \\
\hline Outros & 13 & 21,7 \\
\hline
\end{tabular}

A Figura 1 mostra as doenças apresentadas durante a gestação das lactantes avaliadas no Banco de Leite de Franca que participaram do presente estudo.

Observou-se que todas as participantes do estudo realizaram o pré-natal em número adequado de consultas, correspondendo a 9 e 10 consultas. 
Figura 1 - Doenças apresentadas durante a gestação (n=60) - Franca, 2011)

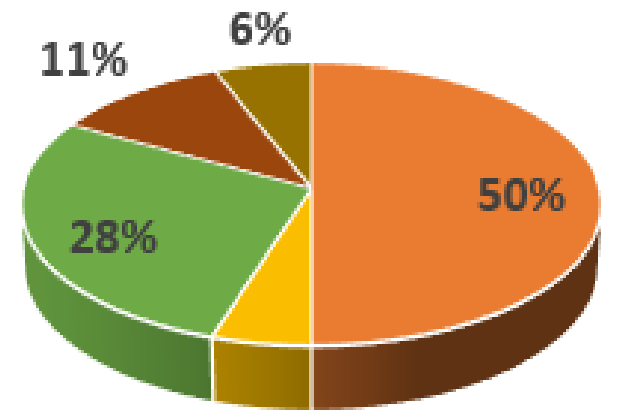

$\mathbf{5 \%}$

- Hipertensão arterial = Epilepsia

- Infecção urinária

- Diabetes mellitus

- Sífilis

As informações nutricionais relacionadas à gestação estão apresentadas na Tabela 2.

Tabela 2 - Características nutricionais relacionadas à gestação ( $(n=60)$ - Franca, 2011

\begin{tabular}{lcc}
\hline Características & $\mathbf{N}$ & $\%$ \\
\hline Orientação Nutricional & 37 & 61,7 \\
$\quad$ Sim & 23 & 38,3 \\
$\quad$ Não & & \\
Ingestão de Sulfato Ferroso & 52 & 86,7 \\
$\quad$ Sim & 8 & 13,3 \\
$\quad$ Não & & \\
Ingestão de Ácido Fólico antes ou durante da & 29 & 48,3 \\
gestação & 31 & 51,7 \\
$\quad$ Sim & & \\
$\quad$ Não & & \\
\hline
\end{tabular}

Sobre os sintomas mencionados durante a gestação, 43 participantes apresentaram náuseas/vômitos $(71,7 \%)$ até o quarto mês. Já a picamalácia foi referida por 24 participantes $(60,0 \%)$, que apresentaram maior desejo de comer alimentos cítricos, como o limão com sal e a manga verde. Outras informações sobre os desejos e aversões alimentares estão expostas na Tabela 3.

Quando questionadas sobre a importância de amamentarem seus filhos, as respostas das mães foram caracterizadas por altruísmo e vontade de ajudar os filhos. Tais características podem ser observadas nas falas a seguir: "[...] Eu amamento meu filho, porque o leite materno é o maior sustento que posso oferecer [...]" (F.C.S). "[...] Amamento meu filho, pois é através do meu leite que vai ajudar na sua formação [...]" (D.A.S). "[...] Porque se eu não amamentar, ele não sobrevive [...]” (E.L). 
Tabela 3 - Aversões e desejos alimentares citados pelas participantes do estudo (n=60) - Franca, 2011.

\begin{tabular}{lcc}
\hline Características & $\mathbf{N}$ & $\%$ \\
\hline Aversões citadas pelas gestantes & & \\
Carnes em geral & 11 & 42,3 \\
Refrigerante & 3 & 11,5 \\
Feijão & 2 & 7,7 \\
Outros alimentos*1 & 14 & 53,8 \\
Desejos citados pelas gestantes - aumento da produção & & \\
láctea & & \\
Canjica & 13 & 41,9 \\
Leite com goiabada & 6 & 19,4 \\
Leite & 3 & 9,7 \\
Outros Alimentos*2 & 9 & 29,0 \\
\hline
\end{tabular}

*1 Outros alimentos: salsicha, doce, charuto, mortadela, sanduíches, pizza, ovo, leite, feijoada, doces, café e fritura.

*2 Outros alimentos: cerveja preta, milho, frango, marmelada, água, sopa de fubá, fígado e couve.

As mães também associavam a amamentação com a proteção aos bebês, como visto nas falas: "[...] Porque o meu leite vai evitar doenças no meu filho [...]" (J.C.N) ou "[...] Eu amamento porque é a única coisa que posso fazer para dar uma vida saudável [...]"(D.A.A).

Das 60 participantes que frequentavam o $\mathrm{BLH}, 35$ estavam em aleitamento materno exclusivo (58\%), 10 estavam em aleitamento materno associado ao uso de fórmulas infantis e 15 em uso apenas de fórmulas infantis. Os motivos pelos quais as mães relataram que não estavam amamentando $(n=15)$ foram pelo parto recente e estarem aguardando a descida do leite $(n=4)$ ou por não poderem estar junto aos bebes prematuros, os quais se encontravam no centro de terapia intensiva (CTI). Elas estavam no BLH exatamente para receber apoio e orientações ao estímulo à lactação, enquanto aguardavam seus filhos saírem do CTI para iniciarem a amamentação.

Em relação à introdução de outros alimentos, 55 participantes (97\%) estavam com bebês recém-nascidos de no máximo 30 dias e, portanto, sem introdução de outros alimentos. Restaram então 5 mães, das quais 3 tinham bebês com 3 a 4 meses e 2 tinham filhos com 1 ano de idade. Estas 5 mulheres introduziram água, chás e papas de fruta e salgada antes de dois meses de idade dos bebês.

A respeito dos motivos da procura pelo $\mathrm{BLH}$, a maior parte das mães entrevistadas procuraram o serviço para o estímulo de produção de leite e para a doação de leite e alívio do ingurgitamento. Duas mães procuraram o serviço por conta de mastite e os 
outros motivos evidenciados foram traumas, rachaduras, bico invertido e pega inadequada.

Com relação ao tempo de acompanhamento no banco de leite a média foi de 7 dias, sendo que apenas uma participante necessitou de um período maior, sendo atendida por 22 dias.

\section{DISCUSSÃO}

A respeito da caracterização das participantes do estudo, observou-se que a maioria era de mulheres adultas jovens e casadas. Um estudo que propôs estudar as particularidades das doadoras de leite em um BLH em Cuiabá-MT encontrou dados semelhantes quanto a idade média e o estado civil dessas mulheres ${ }^{10}$.

As pesquisas sugerem que o apoio conjugal possa ser um diferencial encorajador para a doação de leite humano, uma vez que os dados mostram que as mulheres doadoras são na maioria casadas ou em união estável ${ }^{11,12}$.

A maior parte das mães no presente estudo não possuía trabalho formal, apresentava renda familiar suficiente para a compra de alimentos e era primípara de filhos não planejados e nascidos majoritariamente por cesárea. Estudiosos apontam que o número de partos por cesárea tem crescido exponencialmente nos últimos anos e que boa parte destes procedimentos é feita sem que haja uma necessidade médica real. De 1970 até 2008 este aumento chegou a quase 50\%, sendo mais evidente ainda entre os hospitais privados ${ }^{13}$. Vale ressaltar que a cesariana pode ser o fator de risco mais consistente para a não amamentação na primeira hora de vida, retardando o contato da mãe com o filho e desestimulando este processo ${ }^{1 / 4}$.

Sobre os dados gestacionais, notou-se a presença de doenças como a hipertensão arterial e o diabetes mellitus neste período. Instituições renomadas apontam que o aumento da pressão e da glicemia durante a gravidez são problemas que conferem risco à gestação e aumentam a mortalidade materna ${ }^{15,16}$. Sabe-se que os cuidados durante a gestação, evitando o ganho de peso excessivo, podem contribuir para a diminuição do desenvolvimento de alterações pressóricas e glicêmicas e, por isso, a realização do pré-natal é considerada primordial ${ }^{17}$.

Apesar de todas as mulheres terem realizado o pré-natal suficiente quanto ao número de consultas preconizadas pelo Ministério da Saúde ${ }^{18}$, este não pareceu exercer alguma diferença quanto a prática de doação de leite. Pesquisadores que também encontraram um número suficiente de consultas pré-natal pela maioria das 
mulheres entrevistadas não encontraram correlação estatisticamente significativa com o recebimento de orientação acerca da doação de leite humano ${ }^{19}$.

Além disso, algumas mulheres enquanto gestantes não foram suplementadas e não receberam orientação nutricional. A ausência de suplementação contraria as recomendações da Organização Mundial de Saúde, que orienta a oferta dos micronutrientes como forma profilática ${ }^{20}$. Já a respeito da falta de instruções sobre a alimentação, achados evidenciam que algumas recomendações nutricionais são passadas verbalmente às gestantes, mas poucas vezes são registradas ou prescritas. É importante destacar que o profissional nutricionista poucas vezes integra a equipe que atende essas gestantes para contribuir com as orientações nutricionais ${ }^{21}$.

O nutricionista tem papel importante não só na indicação de uma alimentação adequada às necessidades da gestação, mas também na minimização de sintomas corriqueiros deste período, como as náuseas, vômitos e até mesmo na elucidação de dúvidas sobre os desejos alimentares neste período. Muitas crenças relacionadas ao consumo alimentar na gravidez devem ser desmistificadas para que não comprometa a oferta nutricional adequada e segura ao bebê $\hat{e}^{22,23}$.

No presente estudo os principais sintomas apresentados durante a gestação foram: náuseas, vômitos e picamalácia. O distúrbio da picamalácia foi predominantemente caracterizado no presente estudo pelo desejo de consumir frutas com sal, dado semelhante ao encontrado por Ayeta e colaboradores $^{24}$ em seu estudo. Estes pesquisadores afirmam que a ingestão de itens não alimentícios ou de combinações alimentares inusitadas, denominada como picamalácia, possa estar associada à tentativa de aliviar os próprios sintomas digestivos, bem como a ansiedade e o estresse. Eles ainda reforçam que as práticas alimentares inusitadas devam ser investigadas durante $\mathrm{o}$ atendimento pré-natal para que se adotem medidas psicológicas e nutricionais, evitando riscos à mãe ao concepto ${ }^{24}$.

Sobre as demais preferências e aversões alimentares, destacou-se na presente pesquisa a recusa pelas carnes em geral. Resultados similares foram vistos por Quintanilha e Menezes, que identificaram uma recusa a este grupo alimentar associada não só ao paladar, mas também ao cheiro dos alimentos ${ }^{25}$. Os demais alimentos evitados pelas entrevistadas parecem estar associados àqueles que realmente não devem ser consumidos em quantidades elevadas pelas gestantes ou por qualquer outro indivíduo, como o refrigerante. Quintanilha e Menezes também 
observaram que as gestantes passaram a preferir sucos ao invés das bebidas doces gaseificadas e acreditam que este tipo de escolha é influenciado por lógicas da biomedicina, da cultura, da economia, da estética e da mídia ${ }^{25}$. Já os alimentos mais desejados estiveram relacionados aos mitos alimentares associados à produção de leite, que há anos vêm sendo reportados na literatura ${ }^{26}$.

A respeito da alimentação dos lactentes, todas as mães descreveram a importância da amamentação como forma de garantirem saúde e proteção aos seus filhos e, por isso procuraram o $\mathrm{BLH}$, buscando ajuda para manterem ou iniciarem este processo. Muitas mães chegaram até o serviço com problemas de ingurgitamento, mastite e fissuras no bico do peito. Torquato e colaboradres também encontraram estas mesmas queixas em um estudo descritivo e transversal com 135 nutrizes com características semelhantes às do presente estudo ${ }^{27}$.

Ainda assim, deve-se destacar o uso de fórmulas infantis e a oferta precoce de alimentos aos bebês que foram verificados na presente pesquisa e refletem um cenário desfavorável, mas ainda comum. Um estudo realizado com 700 lactentes com mediana de idade de 5,5 meses também encontrou a introdução antecipada de água, fórmula infantil, chá e suco. Os autores chamam a atenção ainda para a introdução precoce de produtos ultraprocessados, como engrossantes e biscoitos, que podem antecipar a interrupção do aleitamento materno ${ }^{28}$.

Para que as intercorrências relacionadas à mãe e ao bebê não coloquem em risco a amamentação, os estudiosos ressaltam a importância de um acompanhamento prénatal adequado quanto às orientações de ordenha das mamas, pega e suç̧ão do bebê. Além disso, os profissionais de saúde devem ser os principais estimuladores da amamentação e doação do leite materno ${ }^{29}$.

\section{CONCLUSÕES}

Concluiu-se que as mulheres que procuraram o BLH da Santa Casa de Franca eram majoritariamente jovens adultas que haviam tido seus filhos por cesárea e que apresentaram preferências alimentares relacionadas aos mitos de lactogênese.

Apesar de todas as nutrizes terem realizado o pré-natal adequado em quantidade de consultas, estas ainda assim não receberam todas as prescrições nutricionais necessárias e podem ter apresentado problemas no processo da amamentação pela orientação insuficiente quanto à amamentação. No entanto, todas reconheceram a 
importância da amamentação e procuraram auxílio no BLH para iniciarem ou manterem esta prática.

Esta pesquisa enaltece a importância do $\mathrm{BLH}$, que não apenas possibilita o leite às crianças que não puderam ser amamentadas por suas mães, mas também por proporcionar instrução e apoio às lactantes, além do incentivo ao aleitamento materno.

\section{Conflitos de interesse}

Os autores do presente estudo não possuem conflitos de interesse.

\section{REFERÊNCIAS}

1. Maldonado MTP. Psicologia da Gravidez. 1a ed. Petrópolis: Vozes; 1997.

2. Brasil. Ministério da Saúde. Pré-natal e puerpério: atenção qualificada e humanizada: manual técnico. Brasília: MS; 2006.

3. Brasil. Ministério da Saúde. Secretaria de Atenção à Saúde. Departamento de Atenção Básica. Saúde da criança: aleitamento materno e alimentação complementar / Ministério da Saúde, Secretaria de Atenção à Saúde, Departamento de Atenção Básica. - 2. ed. - Brasília: Ministério da Saúde, 2015. 184 p.: il. - (Cadernos de Atenção Básica; n. 23).

4. Amaral LJX, Sales SS, Carvalho DPSRP, Cruz GKP, Azevedo IC, Ferreira Júnior $M A$. Fatores que influenciam na interrupção do aleitamento materno exclusivo em nutrizes. Rev Gaúcha Enferm. 2015;36(esp):127-34.

5. Melo AM. Avaliação da mamada em recém-nascidos prematuros [dissertação] Recife (PE): Universidade Federal de Pernambuco; 2008.

6. Maia PRS, Almeida JAG, Novak FR, Silva DA. Rede Nacional de Bancos de Leite Humano: gênese e evolução. Rev. Bras. Saúde Matern. Infant. 2006; 6(3):285292.

7. Rocha ATS, Lira AYA, Malta DGB, Leitão LP, Mendes CKTT. A importância dos bancos de leite humano na garantia do aleitamento materno. Rev. Ciênc. Saúde Nova Esperança. 2016;14(2):1-8.

8. Brasil. Ministério da Saúde. Campanha incentiva doação de leite materno. Disponível em: http://portalms.saude.gov.br/noticias/agenciasaude/43263-campanha-incentiva-doacao-de-leite-materno.

9. Brasil. Agência Nacional de Vigilância Sanitária. Banco de leite humano: funcionamento, prevenção e controle de riscos / Agência Nacional de Vigilância Sanitária. - Brasília: Anvisa, 2008.160 p.

10. Silva ES, de Jesus LE, dos Santos EB, de Castro NA, Fonseca LB. Doação de leite materno ao banco de leite humano: conhecendo a doadora. Demetra. 2015;10(4);879-889.

11. Fonseca-Machado MOF, Parreira BDM, Dias FA, Costa NS, Monteiro JCS, Gomes-Sponholz FG. Caracterização de nutrizes doadoras de um banco de leite humano. Ciência Cuidado e Saúde 2013.12(3):529-538. 
12. Silva PLN, Jorge JCT, Fonseca JR, Pereira ACA, Oliveira VGR. Perfil das mães doadoras de um banco de leite humano. Revista enfermagem UFPE [Internet]. $2013 ; 7(7): 4635-4640$.

13. Victora CG, Aquino EM, Leal MC, Monteiro CA, Barros FC, Szwarcwald CL. Maternal and child health in Brazil: progress and challenges. Lancet. 2011;377(9780):1863-76.

14. Esteves TMB, Daumas RP, de Oliveira MIC, Andrade CAF, Leite IC. Fatores associados à amamentação na primeira hora de vida: revisão sistemática. Rev Saúde Pública. 2014;48(4):697-703.

15. World Health Organization. WHO recommendations for prevention and treatment of pre-eclampsia and eclampsia. Geneva: World Health Organization; 2011.

16. International Association of Diabetes and Pregnancy Study Groups. Recommendations on diagnosis and classification of hyperglycemia in pregnancy. Diabetes Care. 2010;33(3):676-82.

17. de Oliveira ACM, Graciliano NG. Síndrome hipertensiva da gravidez e diabetes mellitus gestacional em uma maternidade pública de uma capital do Nordeste brasileiro, 2013: prevalência e fatores associados. Epidemiol. Serv. Saúde. 2015;24(3):441-451.

18. Brasil. Ministério da Saúde. Secretaria de Atenção à Saúde. Departamento de Atenção Básica. Atenção ao pré-natal de baixo risco. Brasília: Ministério da Saúde, 2012. 318 p. (Série A. Normas e Manuais Técnicos).

19. Muller KTC, Souza AIP, Cardoso JMF, Palhares DB. Conhecimento e adesão à doação de leite humano de parturientes de um hospital público. INTERAÇÕES. 2019;20(1):315-326.

20. Organização Mundial da Saúde (OMS). Diretriz: Suplementação intermitente de ferro e ácido fólico em gestantes não anêmicas. Genebra: OMS; 2013.

21. Niquini Rp, Bittencourt SA, Lacerda EMA, Saunders C, Leal MC. Avaliação do processo da assistência nutricional no pré-natal em sete unidades de saúde da família do Município do Rio de Janeiro. Ciência \& Saúde Coletiva. 2012; 17(10):2805-2816.

22. Gomes MRT, Silva LT, Salamoni RM. Investigação dos tabus e crenças alimentares em gestantes e nutrizes do hospital regional de Mato Grosso do SulRosa Pedrossian. Ciências Agrárias, Biológicas e da Saúde. 2011;15(6):121-133.

23. Gomes CB, Vasconcelos LG, Cintra RMGC, Dias LCGD, Carvalhaes MABL. Hábitos alimentares das gestantes brasileiras: revisão integrativa da literatura. Ciênc. saúde coletiva. 2019; 24(6):2293-2306.

24. Ayeta AC, da Cunha ACB, Heidelmann SP, Saunders C. Fatores nutricionais e psicológicos associados com a ocorrência de picamalácia em gestantes. Rev Bras Ginecol Obstet. 2015; 37(12):571-7.

25. Karina Elias de Quintanilha; Maria Fátima Garcia de Menezes. Desejos e aversões alimentares: a visão de gestantes usuárias do serviço de obstetrícia da Policlínica Piquet Carneiro. CERES. 2010; 5(2); 73-88.

26. Ichisato SMT, Shimo AKK. Aleitamento materno e as crenças alimentares. Rev Latino-am Enfermagem. 2001;9(5):70-6. 
27. Torquato RC, Silva VMGN, Lopes APA, Rodrigues LN, da Silva WCP, Chaves EMC. Perfil de nutrizes e lactentes atendidos na Unidade de Atenção Primária de Saúde. Esc Anna Nery. 2018;22(1):1-6.

28. Moreira LCQ Oliveira EB, Lopes LHK, Bauleo ME, Sarno F. Introdução de alimentos complementares em lactentes. Einstein. 2019;17(3)1-6.

29. de Meneses TMX, de Oliveira MIC, Boccolini CS. Prevalência e fatores associados à doação de leite para postos de recebimento de leite humano de unidades básicas de saúde. J. Pediatr. 2017;93(4):382-388.

Submissão: 04/09/2018

Aprovação: 15/10/2020 